\title{
ANÁLISE BIBLIOMÉTRICA DA ESPECTRORRADIOMETRIA E GEOESTATÍSTICA DE SOLOS: UMA DISCUSSÃO EM 10 ANOS
}

\author{
BIBLIOMETRIC ANALYSIS OF SPECTRORADIOMETRY AND SOIL GEOSTATISTICS: \\ A 10-YEAR DISCUSSION
}

\author{
Jonathas Jesus dos SANTOS ${ }^{1}$; Washington de Jesus Sant'Anna da FRANCA-ROCHA ${ }^{2}$; \\ Gustavo Macedo de Mello BAPTISTA ${ }^{3}$; Deorgia Tayane Mendes de SOUZA² ${ }^{2}$ Ardemirio de \\ Barros SILVA ${ }^{2}$; Sarah Moura Batista dos SANTOS ${ }^{3}$ \\ ${ }^{1}$ Universidade de São Paulo - USP. Departamento de Geografia. Avenida Professor Lineu Prestes, 338. Cidade Universitária, São \\ Paulo - SP. Email: jonathas@usp.br \\ ${ }^{2}$ Universidade Estadual de Feira de Santana. Avenida Transnordestina, s/n, Novo Horizonte - BA. \\ E-mails: wrocha@uefs.br; deorgiasouza.geo@gmail.com; abarros@uefs.br; \\ ${ }^{3}$ Universidade de Brasília, Instituto de Geociências. Campus Universitário Darci Ribeiro. Brasília- DF. E-mail: gmbaptista@unb.br \\ ${ }^{4}$ Universidade do Minho - UMinho, Departamento de Geografia. Campus de Azurém, Guimarães - Portugal. \\ -mail: saamoura@gmail.com

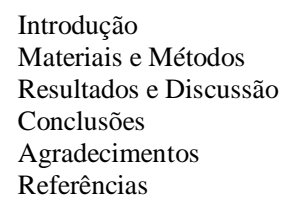

RESUMO - A propagação e disponibilidade de fontes e recursos de informação em formato digital tem favorecido o desenvolvimento de indicadores importantes, como exemplo das pesquisas bibliométricas para a mensuração da atividade científica. Nessa perspectiva, pretende-se analisar multitemporalmente as publicações em periódicos científicos sobre espectrorradiometria e solos, geoestatística de solos, em uma escala temporal de 10 anos. Nesse sentido, utilizou-se a plataforma SCOPUS para a análise, configurando os dados de busca, selecionando somente os artigos, o limite de busca por ano e o limite de campo de conhecimento. Logo depois, as informações gerais de todos os periódicos incluindo país de publicação, nome dos autores, coautores, citações, entre outros, foram exportados e estabeleceu-se as redes semânticas no programa VOSviewer®, selecionando-se o período de 2007 a 2017 . Observou-se um crescimento do número de publicações dos dois temas pesquisados ao longo do tempo, os países que mais publicaram sobre espectrorradiometria e solos são os EUA e o Reino Unido e, sobre geoestatística de solos a China e o Brasil.

Palavras-Chave: Análise Multitemporal, Densidade de publicações, Redes semânticas.

\begin{abstract}
The increase of bibliometric surveys in the measurement of scientific activity has favored the development of important indicators. In this perspective, we intend to analyze the publications in scientific journals on spectroradiometry and soils, geostatistics and soil fertility on a 10-year time scale. Therefore, the SCOPUS platform was used for the analysis, configuring the search data, selecting only the articles, the search limit per year and the knowledge field limit. Subsequently, the general information of all journals including country of publication, authors' names, co-authors, citations, among others, were exported and semantic networks were established in the VOSviewer® program, selecting the period from 2007 to 2017 . There was an increase in the number of publications of the two themes surveyed over time, the country that most published on spectroradiometry and soils is the USA, and on geostatistics and soil fertility in China and Brazil.
\end{abstract}

Keywords: Multitemporal Analysis, Density of publications, Semantic networks.

\section{INTRODUÇÃO}

A utilização da bibliometria tem favorecido o desenvolvimento de indicadores quantitativos e ferramentas importantes. O aumento de sua utilização na mensuração da atividade científica se deve, em parte, à propagação e disponibilidade de fontes e recursos de informação em formato digital. Estas fontes informatizadas tornaram possível o acesso e a manipulação de grandes volumes de dados (Lima et al., 2010).

$\mathrm{Na}$ atualidade, duas técnicas de difusão recente no meio científico despertam atenção para a análise bibliométrica: espectrorra- diometria de solos e a geoestatística de solos. A espectrorradiometria ou espectroscopia de reflectância é uma técnica avançada dentro do sensoriamento remoto muito útil na avaliação de variações pontuais do solo, podendo ser utilizada para identificar as classes e propriedades de solos com grande precisão (Genú et al., 2013; Demattê \& Terra, 2014; Bonett et al., 2015).

O uso de sensores terrestres na aquisição de dados espectrais de solos tem favorecido a caracterização e o avanço de técnicas de quantificação de atributos (Bellinaso et al., 
2010). Essa técnica pode ser muito útil na avaliação de variações pontuais do solo - em profundidade (de diferentes camadas e, ou horizontes) devido à interação da luz com propriedades do solo refletindo dados intrínsecos relacionados com eles (Demattê \& Terra, 2014). Também pode ser utilizada como alternativa para quantificação de atributos como matéria orgânica do solo (Dotto et al., 2014), para caracterização granulométrica de amostras de solos de diferentes classes texturas (Franceschini et al., 2013; Dotto et al., 2014), para predição de atributos químicos e físicos dos solos (Chang et al., 2001), entre outros.

A Geoestatística é uma ferramenta que emprega a definição de variáveis regionalizadas na avaliação de variabilidade espacial por meio da extração e organização espacial dos dados disponíveis de acordo com a semelhança entre pontos vizinhos georreferenciados (Grego et al., 2014). Nesse sentido, a espacialização de dados de solo, por meio da geoestatística, tem sido utilizada em vários tipos de pesquisas na atualidade. A exemplo do estudo da variabilidade espacial dos atributos dos solos para que se possa aprimorar as opções de manejo e melhorar a rentabilidade do sistema de produção (Bernardi et al., 2014).

Além disso, o conhecimento das distintas variáveis pode ser uma ferramenta essencial para o desenvolvimento de projetos relacionados a métodos de interpolação e metodologias estatísticas para validar a correlação entre as características referentes aos solos (Ronquim, 2010). Nessa perspectiva, este trabalho tem o objetivo de analisar multitemporalmente as publicações em periódicos científicos sobre espectrorradiometria de solos e geoestatística de solos, em uma escala temporal de 10 anos.

\section{MATERIAIS E MÉTODOS}

Para a análise bibliométrica da espectrorradiometria de solos, geoestatística de solos, utilizou-se a plataforma SCOPUS, tratando-se uma base de dados originalmente europeia pertencente a editora Elsevier. A plataforma possui atualmente uma ampla coleção de periódicos científicos, sendo uma das maiores bases de dados de periódicos do mundo, incluindo artigos publicados a partir de 1966 (Falagas et al., 2008; Puccini et al., 2015).

Para a pesquisa dentro da plataforma, configurou-se os dados específicos, definindo os assuntos de interesse, selecionando somente os periódicos científicos, o limite de busca por ano e o limite de campo de conhecimento. Com os resultados da pesquisa, observou-se os dados analíticos em gráficos em escala global dentro da plataforma: os países, autores, campos de conhecimento que mais publicaram sobre o tema, os tipos de documentos publicados, além da evolução temporal das publicações ao longo dos anos.
Logo depois, as informações gerais de todos os artigos incluindo país de publicação, nome dos autores, coautores, citações, entre outros, foram exportados em formato csv para serem estabelecidas as redes semânticas no programa VOSviewer®. No software, as redes foram estabelecidas por países que mais publicaram o tema espectrorradiometria de solos e geoestatística dos solos, com as conexões entre eles em rede e com a visualização em diferentes escalas.

A análise da evolução temporal de dez anos de publicação consistiu na seleção do período de 2007 a 2017, tratando-se de um tempo mais recente para a avaliação da evolução das publicações. Nessa perspectiva, estabeleceu-se um gradiente entre o período com cores em lilás próximo de 2007 , em azul e verde entre 2010 e 2014, em amarelo entre 2016 e 2017, permitindo a interpretação dos países que já publicaram o tema desde 2007 e os países mais recentes na publicação do tema.

\section{RESULTADOS E DISCUSSÃO}

Os estudos sobre a espectrorradiometria de solos têm aumentado de forma exponencial desde o início dos anos 2000, fato identificado na evolução temporal das publicações envolvendo o tema no mundo (Figura 1).

Os primeiros trabalhos, avaliando o comportamento espectral dos alvos mediante sua resposta espectral, foram realizados nas décadas de 60 e 70 com Obukhov \& Orlov (1964); Bowers \& Hanks (1965); Hunt \& Salisbury
(1970), ao longo desse período, foram surgindo várias ramificações (Bellinaso et al., 2010; Genú et al., 2013, Demattê \& Terra, 2014).

Como exemplo da evolução das ramificações dos estudos de solos por meio da espectrorradiometria de acordo com a Nasa (1987), tem-se as investigações sobre a estrutura (Obukhov \& Orlov, 1964), óxidos de ferro e mineralogia (Bowers \& Hanks 1965; Baumgardner \& Stoner, 1982) a matéria orgânica (Myers \& Allen, 1968), 
tamanho e distribuição das partículas (Karmanov, 1970), umidade (Planet, 1970; Bowers \& Smith, 1972), e material parental para a reflectância de solos naturais (Stoner et al., 1980).

De 2007 a 2017 com as crescentes demandas de dados sobre solos, vários estudos se empenharam no desenvolvimento e aprimoramento de técnicas de avaliação de atributos por espectrorradiometria, a exemplo de Cezar et al. (2013) que estimou os teores de silte, areia, argila, soma de bases, matéria orgânica e ferro total em amostras de solo, a partir de sua energia refletida por meio de regressão linear múltipla; Romagnoli et al. (2014) que utilizou o método PLSR (ParLeS) e a regressão linear múltipla com seleção de variáveis Stepwise (SAS), para predição do carbono orgânico do solo por meio de dados de reflectância; Demattê et al., (2016) que estudou a qualidade dos modelos de predição de atributos dos solos.

Analisando a rede semântica, dentre os países que mais publicaram sobre o tema espectrorradiometria de solos de 2007 a 2017, os Estados Unidos, Canadá e Reino Unido destacam-se desde 2007 (Figura 2).

Tal fato pode estar associado a língua inglesa predominante nos três países e as publicações por filiações de outros países, sendo que os Estados Unidos foi o país pioneiro no desenvolvimento da espectrorradiometria. Em meados de 2010 a Alemanha, Itália, França e Nova Zelândia também ganham destaque e logo depois em 2012 o Brasil, China e demais países tem publicações envolvendo o tema (Figura 2). Observando a densidade de periódicos sobre o tema (Figura 3), verifica-se que que nesse período o maior número de publicações é dos Estados Unidos, Brasil, Alemanha e China.

Documents by year

50

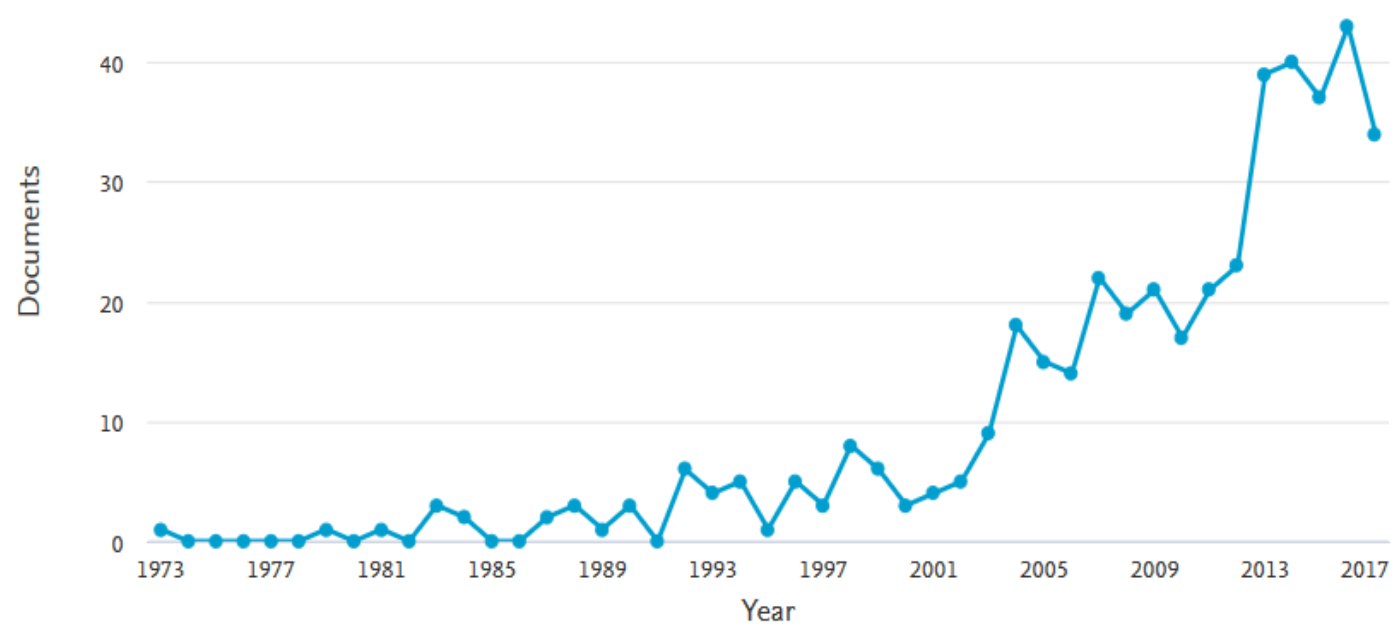

Figura 1 - Publicações em periódicos científicos no mundo sobre espectrorradiometria e solos de 1973 a 2017 . Observase uma maior ascensão de publicações no início dos anos 2000. Fonte: SCOPUS, 2018.

Observando o gráfico temporal de publicações científicas no mundo sobre geoestatística de solos, é verificável que o número de publicações envolvendo o tema tem aumentado desde a década de 90 (Figura 4). A partir dessa década os estudos sobre a variabilidade espacial das propriedades dos solos torna-se uma questão fundamental para os pesquisadores (Vieira, 2000).

Entretanto, o crescimento do número de publicações está relacionado ao desenvolvimento de três fatores, o primeiro deles é o desenvolvimento da informática, que permitiu a inferência estatística em grandes conjuntos de dados pedométricos, por exemplo, taxonomia numérica de solos do início da década de 1960 e geoestatística a partir de meados da década de 1970, bem como modelos de simulação de processos do solo (Rossiter, 2018).

Um segundo fator é o crescimento da rede. Isso facilitou a colaboração entre pedometristas, comunicação rápida com periódicos, programação e publicação colaborativa e fácil acesso a recursos. Um terceiro fator é a disponibilidade de armazenamento on-line de grandes conjuntos de dados, especialmente de dados abertos, incluindo coberturas SIG e imagens de sensoriamento remoto permitindo que os cientistas individuais realizassem procedimentos complexos (Rossiter, 2018). 


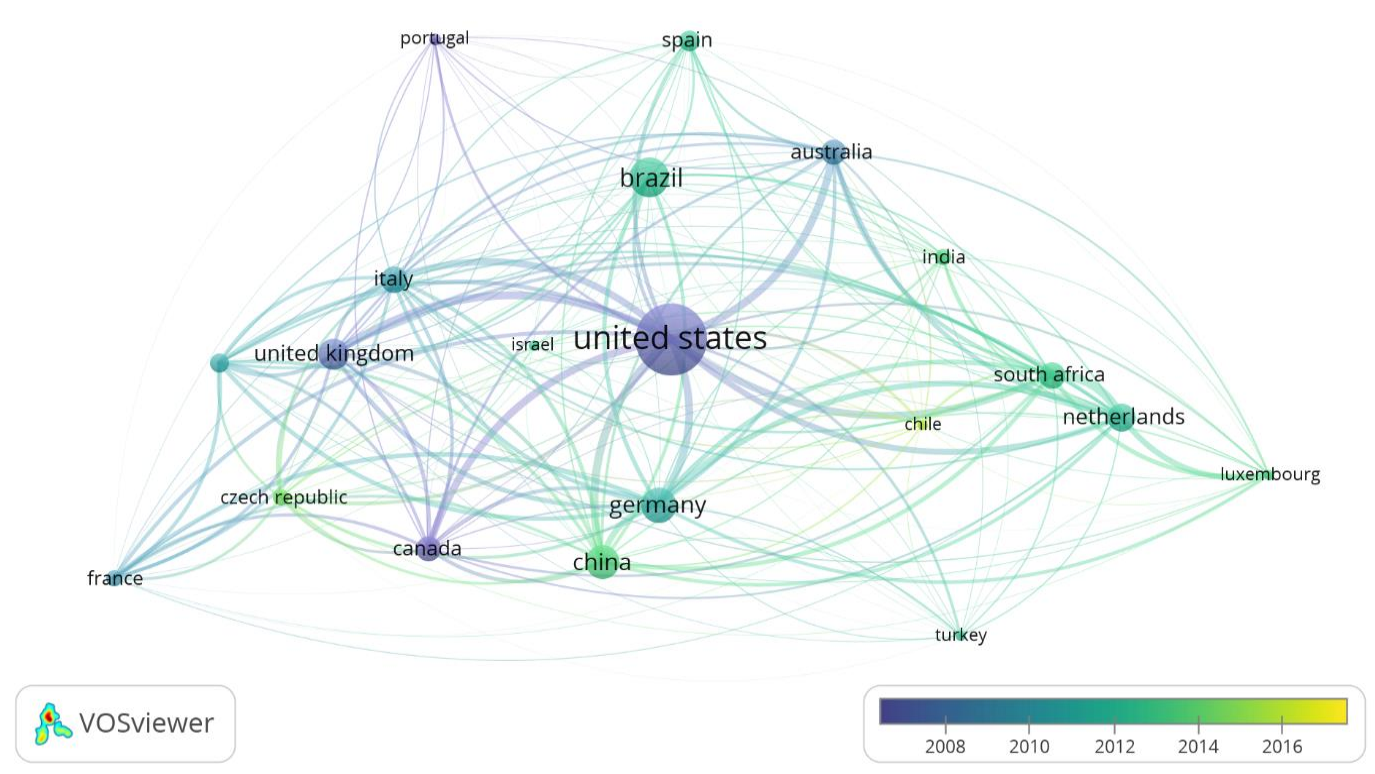

Figura 2 - Países que mais publicaram artigos no mundo com o tema Espectrorradiometria de solos ao longo dos anos 2007 a 2017.

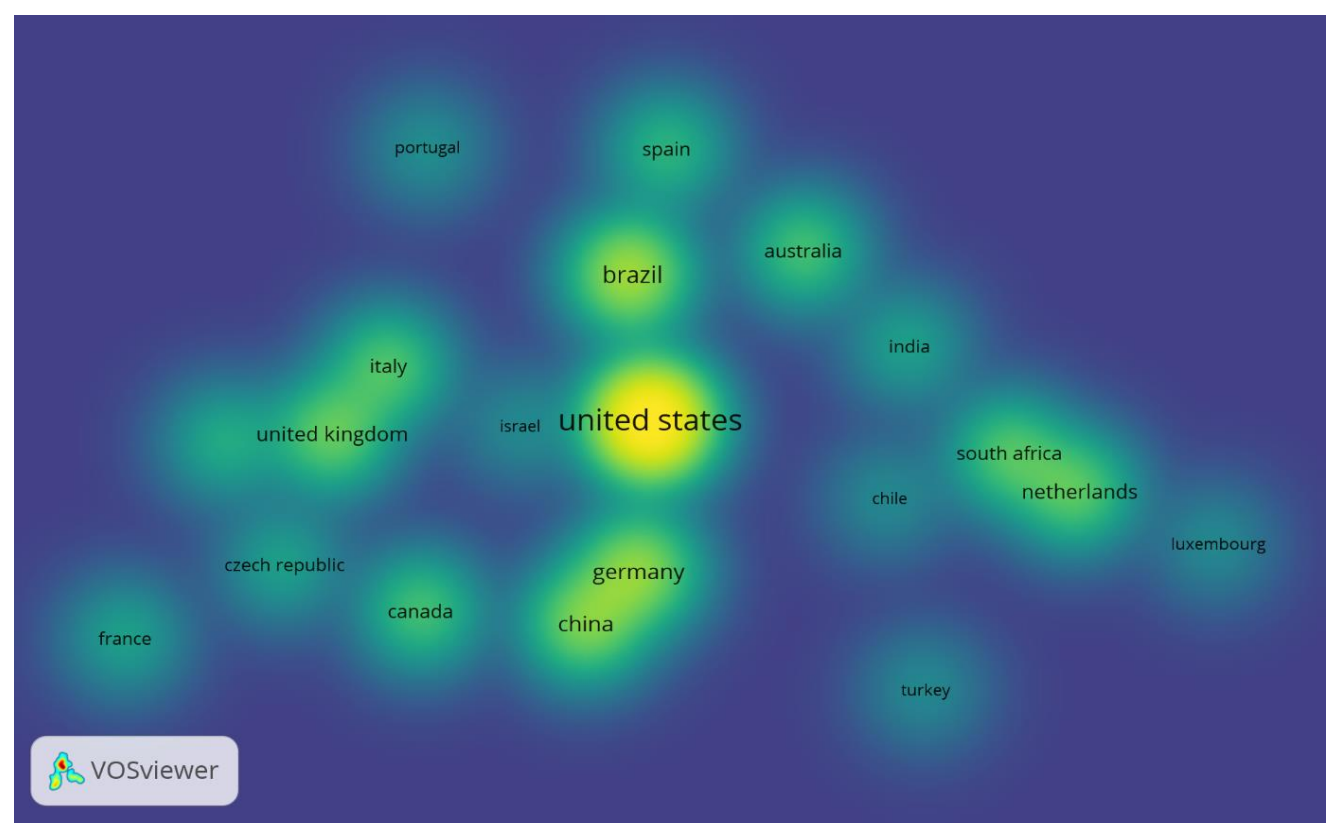

Figura 3 - Densidade de periódicos científicos no mundo envolvendo o tema Espectrorradiometria e solos. As cores em maiores densidades representam os países que mais publicaram o tema. Em contrapartida, as cores com menores densidades os países que publicaram o tema, mas em menor quantidade.

De 2007 a 2017 a Geoestatística dos solos tem sido utilizada em diferentes temas baseando-se na aplicação de tecnologias de maneira localizadas de acordo com a variabilidade espacial, a exemplo da sua utilização para a avaliação da distribuição de elementos maiores nos solos (Wei et al., 2017); na identificação da variabilidade de atributos mineralógicos (Camargo et al., 2008); na agricultura de precisão (Silva \& Lima, 2009; Grego et al., 2014 ; Zonta et al., 2014); na distribuição espacial da umidade do solo (Ávila et al., 2010).
Estudos também sobre a distribuição do carbono orgânico nos solos (Allaire et al., 2012; Cambule et al., 2014; Chen et al., 2015); metais pesados (Silva et al., 2013; González et al., 2014; Li et al., 2015); da matéria orgânica (Leite et al., 2015); no Mapeamento Digital de Solos (Bishop et al., 2015); na avaliação das propriedades dos solos (Sumfieth et al., 2008; Campos et al., 2013; Lima et al., 2014); no mapeamento 3D dos atributos pedológicos (Choi \& Park, 2009, Adhikari et al., 2013), entre outras temáticas de investigação dos solos. 


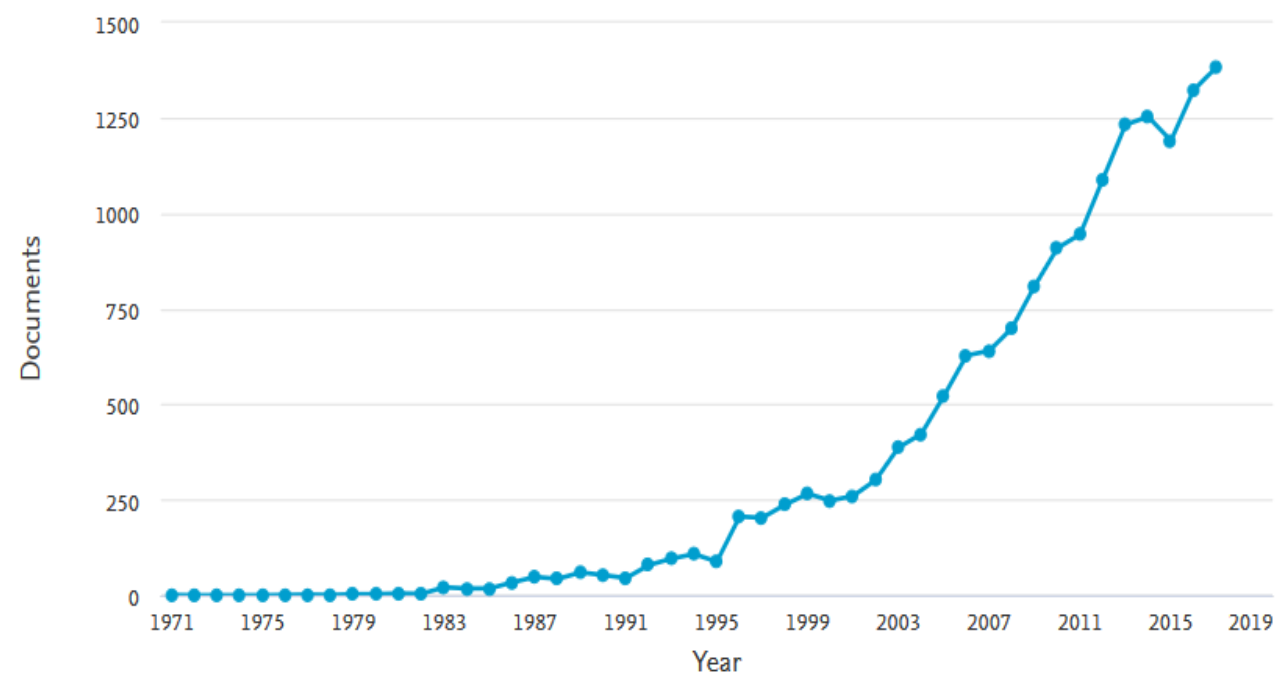

Figura 4 - Publicações em periódicos científicos no mundo sobre geoestatística de solos de 1971 a 2018. Observa-se uma maior ascensão de publicações no final do ano de 1995. Fonte: SCOPUS, 2018.

Analisando a rede de países que publicaram em periódicos científicos envolvendo o tema geoestatística dos solos (Figura 5), é observável que a maior quantidade de publicações envolvendo o tema foi da China e o Brasil, fato também verificado na densidade de publicações (Figura 6).

Entretanto, na análise multitemporal verificase que por filiação entre 2007 e 2008 os países que mais publicaram sobre o tema eram os Estados Unidos, Alemanha e Países Baixos. Em seguida, entre de 2010 a 2012 surge a Itália, Áustria e Venezuela (Figura 5).

Posteriormente, destaca-se a China e o Brasil entre 2012 a 2014, juntamente com a Espanha, Irã, Turquia, Austrália. Entre 2015 a 2017 surgem os países mais recentes em publicações como o Canadá, Croácia, Austrália, Índia e Egito. Nesse contexto, é verificável um crescente interesse pelo tema ao longo do tempo, com novos países pesquisando sobre a temática e novas conexões em redes entre eles.

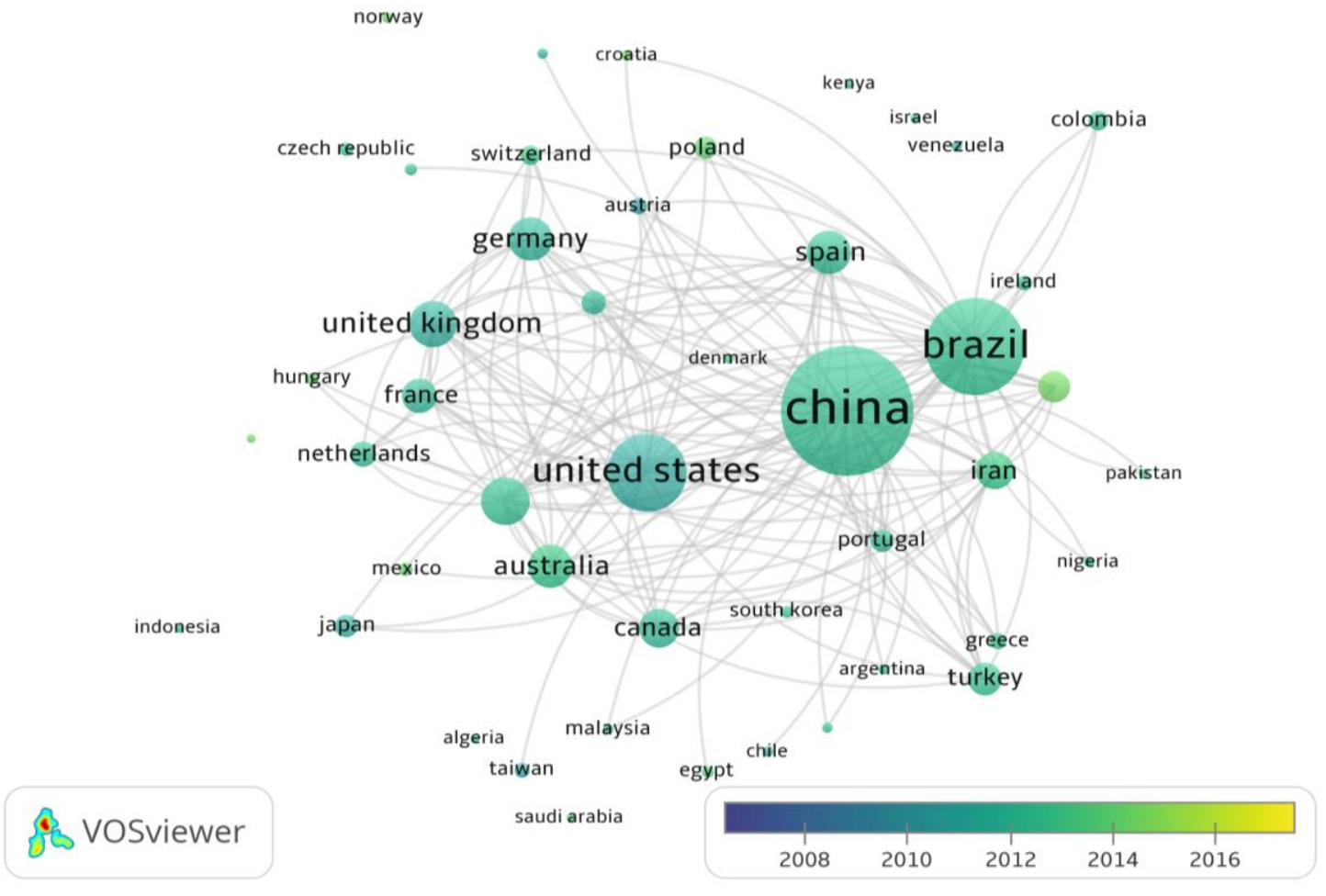

Figura 5 - Países que mais publicaram em periódicos científicos no mundo envolvendo o tema Geoestatística de solos de 2007 a 2017. Os países estão representados em círculos de densidade no qual os maiores círculos possuem mais publicações. As cores de cada círculo estão correlacionadas com a paleta de cores multitemporal de 2007 a 2017. 


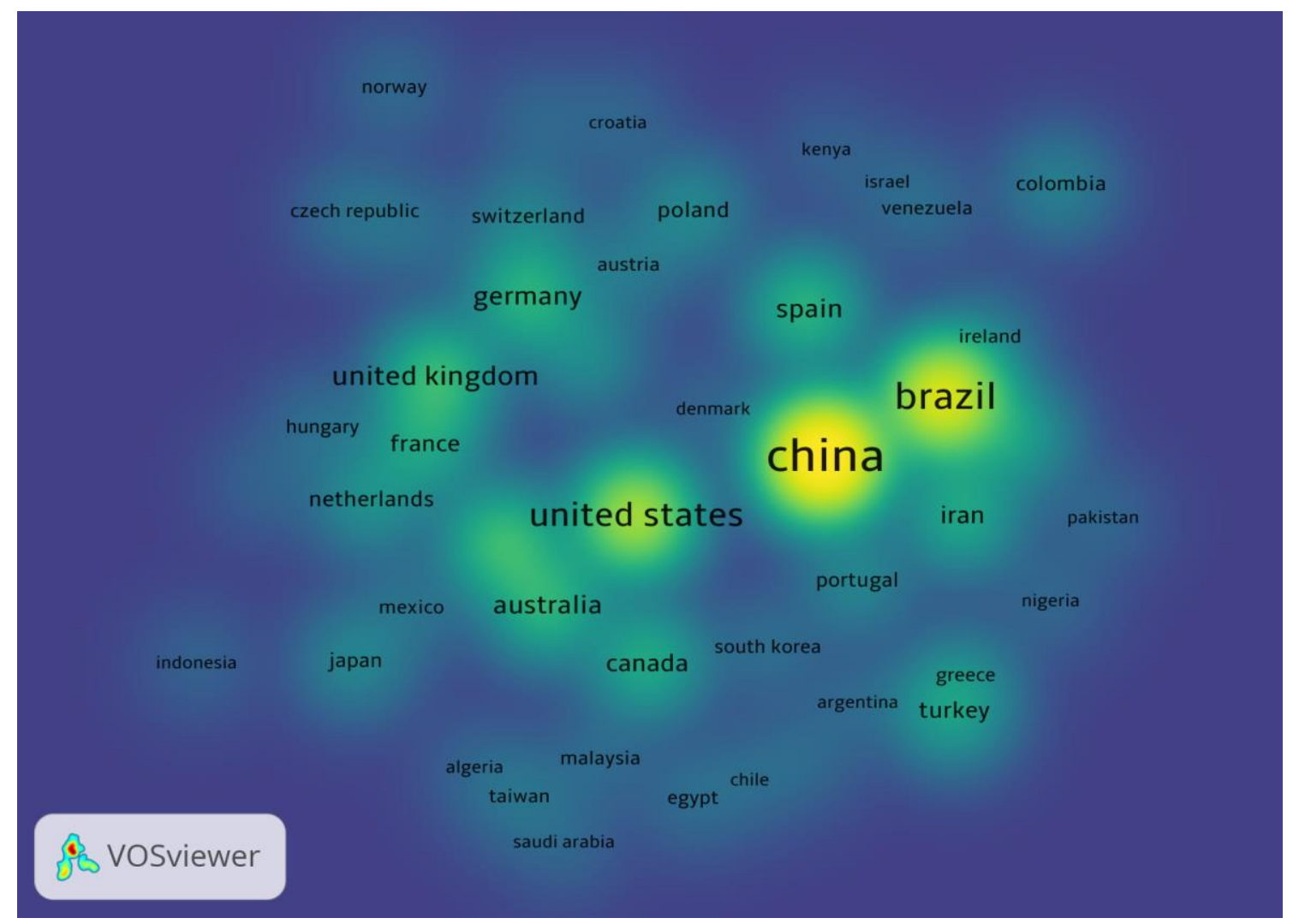

Figura 6 - Densidade de periódicos científicos no mundo envolvendo o tema Geoestatística de solos. As cores em maiores densidades representam os países que mais publicaram o tema. Em contrapartida, as cores com menores densidades os países que publicaram o tema, mas em menor quantidade.

\section{CONCLUSÃO}

A análise bibliométrica sobre a espectrorradiometria de solos, Geoestatística dos solos demonstra um forte crescimento nas pesquisas mundiais sobre os temas em diferentes países entre 2007 e 2017. É verificado também um forte relacionamento em rede entre os países que pesquisam sobre os temas, fato relacionado ao desenvolvimento da informática, possibilitando uma maior parceria e construções de periódicos com coautores de distintos lugares.

As densidades de publicações, revela no primeiro caso uma concentração de publicações nos países pioneiros sobre o assunto, resultante do desenvolvimento e da utilização dos primeiros equipamentos de espectrorradiometria nos países como os Estados Unidos e Reino Unido, Alemanha e Brasil. No segundo caso, a densidade de publicações na China e no Brasil está relacionada com o desenvolvimento da agricultura de precisão para práticas agrícolas.

\section{AGRADECIMENTOS}

Ao Programa de Pós-Graduação Modelagem em ciências da Terra e do Ambiente (PPGM), da Universidade Estadual de Feira de Santana (UEFS). Ao Instituto Nacional em Ciência e Tecnologia em Estudos Interdisciplinares e Transdisciplinares em Ecologia e Evolução (INCT IN-TREE). Ao Projeto de Mapeamento Anual da Cobertura e Uso do Solo no Brasil (MapBiomas). A Coordenação de Aperfeiçoamento de Pessoal de Nível Superior (CAPES).

\section{REFERÊNCIAS}

ADHIKARI, K.; KHEIR, R.B.; GREVE, M.B., BOCHER, P.K.; MALONE, B.P.; MINASNY, B.; MCBRATNEY, A.B.; GREVE, M.H. High-resolution 3-d mapping of soil texture in Denmark. Soil Sci. Soc. Am. J, v. 77, n. 3, p. 860-876, 2013.

ALLAIRE, S.E.; LANGE, S.F.; LAFOND, J.A.; PELLETIER, B.; CAMBOURIS, A.N.; DUTILLEUL, P. Multiscale spatial variability of $\mathrm{CO} 2$ emissions and correlations with physicochemical soil properties. Geoderma, v. 170, p. 251-260, 2012.

ÁVILA, L.F.; DE MELLO, C.R.; DA SILVA, A.M. Continuidade e distribuição espacial da umidade do solo em bacia hidrográfica da Serra da Mantiqueira. Revista Brasileira De Engenharia Agrícola E Ambiental, v. 14, p. 1257-1266, 2010.

BAUMGARDNER, M.F. \& STONER, E.R. Soil mineralogical studies by remote sensing, New Delhi, India, Trans. In: International Soil Congress, 12. Anais... New Delhi: n. 5, p. 419, 1982.

BELLINASO, H.; DEMATTÊ, J.A.M.; ROMEIRO, S.A. Soil Spectral Library and its use in Soil Classification. Revista Brasileira de Ciências dos Solos, v. 34, p. 861-870, 2010. 
BERNARDI, A.C.; RABELLO, L.M.; INAMASU, R.Y.; GREGO, C.R.; ANDRADE, R. G. Variabilidade espacial de parâmetros físico-químicas de solo e biofísicos de superfície em cultivo do sorgo. Campina Grande: Revista Brasileira de Engenharia Agrícola, v. 18, v. 6, p. 663-630, 2014.

BISHOP, T.F.A.; HORTA, A.; KARUNARATNE, S.B.; Validation of digital soil maps at different spatial supports. Geoderma, v. 241-242, p. 238-249, 2015.

BONETT, J.P.; TAMAYO, C.J.H.; LÓPEZ, L.R. Espectroscopia de infrarrojo medio para la estimación de algunas propiedades del suelo. Agronomia Colombiana, n. 33, v. 1, p. 99-106, 2015.

BOWERS, S.A. \& HANKS, R.J. Reflection of radiant energy from soils. Soil Science, Baltimore, v. 100, n. 2, p. 130-138, 1965.

BOWERS, S.A. \& SMITH, S.J. Spectrophotometric determination of soil water content, Soil Science Society of America Journal, v. 36, p. 978, 1972.

CAMARGO, L.A.; JÚNIOR, J.M.; PEREIRA, G.T., HORVAT, R.A. Spatial variability of attributes of an axisol under different relief forms. Revista Brasileira de Ciência dos Solos, v. 32, p. 2279-2288, 2008.

CAMBUlE, A.H., ROSSITER, D.G., STOORVOGEL, J.J., SMALING, E.M.A., Soil organic carbon stocks in the Limpopo National Park, Mozambique: amount, spatial distribution and uncertainty. Geoderma, v. 213, p. 46-56, 2014.

CAMPOS, M.C.C., SOARES, M.D.R., SANTOS, L.A.C., OLIVEIRA, I.A., AQUINO, E.A., Spatial variability of physical attributes in Alfissol under agroforestry, Humaitá region, Amazonas state, Brazil. Revista de Ciências Agraria, v. 56, p. 149-159, 2013.

CEZAR, E.; NANNI, M.R.; DEMATTÊ, J.A.M.; CHICATI, M.L.; OLIVEIRA, R.B. Estimativa de Atributos do Solo por meio de Espectrorradiometria Difusa. Revista Brasileira de Ciência dos Solos, v. 37, p. 858-868, 2013.

CHANG, C.W.; LAIRD, D.A.; MAUSBACH, M.J. \& HURBURGH JUNIOR, C.R. Near-infrared reflectance spectroscopy - principal components regression analyses of soil properties. Soil Science Society of America Journal, v. 65, p. 480-490, 2001.

CHEN, C.; HU, K.; LI, H.; YUN, A.; LI, B.; Three-Dimensional Mapping of Soil Organic Carbon by Combining Kriging Method with Profile Depth Function. Journal Pone, v. 10, n. 6, 2015.

CHOI, Y. \& PARK, H. Integrating GIS and 3D geostatitical methods for geotecnical characterization of soil properties. Engineering Geology for Tomorrow's Cities. Engineering Geology Special Publications, CULSHAW, M. G., REEVES, H.J., JEFFERSON, I.; SPINK, T.W. (ed.), Geological Society, London, 2009.

DEMATTÊ, J.A.M. \& TERRA, F.S. Spectral pedology: A new perspective on evaluation of soils along pedogenetic alterations. Geoderma, v. 217-218, n. 190, 2014.

DEMATTÊ, J.A.M.; BELLINASO, H.; ARAÚJO, S.R.; RIZZO, R.; SOUZA, A.B. Regionalização espectral de solos tropicais na estimativa dos atributos do solo. Revista Ciência Agronômica, v. 47, n. 4, p. 589-598, 2016.

DOTTO, A.C.; DALMOLIN, R.S.D.; PEDRON, F.A.; CATEN, A.T.; RUIZ, L.F.C.; Mapeamento digital de atributos: Granulometria e matéria orgânica do solo utilizando a espectroscopia de reflectância difusa. Revista Brasileira de Ciência dos Solos, v. 38, p.1663-1671, 2014.

FALAGAS, M.E; PITSOUNI, E.I.; MALIETZIS, G. A.; PAPPAS, G. Comparison of PubMed, Scopus, Web of Science, and Google Scholar: strengths and weaknesses. Scientific Databases, Pros and cons. The FASEB Journal, v. 22, 2008.

FRANCESCHINI, M.H.D.; DEMATTÊ, J.A.M; SATO, M.V.; VICENTE, L.E.; GREGO, C.R. Abordagens semi-quantitativa e quantitativa na avaliação da textura do solo por espectroscopia de reflectância bidirecional no VIS- NIR- SWIR. Brasília.
Pesquisa Agropecuária Brasileira, v. 48, n. 12, p. 1569-1582, 2013.

GENÚ, A.M.; DEMATTÊ, J.A.M.; NANNI, M.R. Caracterização e comparação do comportamento espectral de atributos do solo obtidos por sensores orbitais (ASTER e TM) e terrestre (IRIS). Guarapuava-PR. Ambiência, v. 9, n. 2, 2013.

GONZÁLEZ, I.; NEAMAN, A.; RUBIO, P.; CORTÉS, A.; Spatial distribution of copper and $\mathrm{pH}$ in soils affected by intensive industrial activities in Puchuncaví and Quintero, central Chile. Journal of Soil Science and Plant Nutrition, v. 14, p. 943-953, 2014.

GREGO, C.R.; OLIVEIRA, R.P.; VIEIRA, S.R. Geoestatística aplicada a Agricultura de Precisão. In: BERNARDI, A.C.C.; NAIME, J.M.; RESENDE, Á.V.; BASSOI, L.H.; INAMASU, R.Y. (Org.). Agricultura de precisão: resultados de um novo olhar. 2ed.Brasília, DF: Embrapa, v. 1, p. 74-83, 2014.

HUNT, G.R. \& SALISBURY, J.W. Visible and near infrared spectra of minerals and rocks I: Silicate minerals. Modern Geology, London, v. 1, p. 283-300, 1970.

KARMANOV, I.I. Study of soils from the spectral composition of reflected radiation, Sov. Soil Sci., v. 4, p. 226, 1970.

LEITE, L.F.C.; FERREIRA, J.S.; VELOSO, M.E.C.; MOUSINHO, F.E.P.; ROCHA JUNIOR, A.F. Variabilidade espacial das frações da matéria orgânica do solo em área degradada sob recuperação. Revista Brasileira de Engenharia Agrícola e Ambiental, v. 19, n. 4, p. 394-401, 2015.

LI, Y.M.; MA, J.H.; LIU, D.X. Assessment of heavy metal pollution and potential ecological risks of urban soils in Kaifeng city, China Environmental Science, v. 36, n. 3, p. 1037-1044, 2015.

LIMA, R.A.; VELHO, L.M.L.S.; FARIA, L.I.L. Análise bibliométrica da atividade científica da Embrapa Solos (1975-2009). Rio de Janeiro-RJ: Boletim de Pesquisa e Desenvolvimento-Embrapa solos, 2010.

LIMA, G.C.; SILVA, M.L.M.; OLIVEIRA, M.S.; CURI, N.; SILVA, M.A.; OLIVEIRA, A.H. Variabilidade de atributos do solo sob pastagens e mata atlântica na escala de microbacia hidrográfica. Revista Brasileira de Engenharia Agrícola e Ambiental, v. 18, n. 5, p. 517-526, 2014.

MYERS, V.I.; ALLEN, W.A. Electrooptical Remote Sensing Methods as Nondestructive Testing and Measuring Techniques in Agriculture. Issue 9, v. 7, p. 1819-1838, 1968.

NASA. Hiris High resolution Imaging spectrometer: science opportunitles for the 1990s. Earth Observing Systen, v. 2c, 1987.

OBUKHOV, A.I. \& ORLOV, D.S. Spectral reflectivity of the major soil groups and possibility of using diffuse reflection in soil investigation. Soviet Soil Science, Washington, v. 1, p. 174-184, 1964.

PLANET, W.G., Some comments on reflectance measurements of wet soils, Remote Sens. Environ., v. 1, p. 127, 1970.

PUCCINI, L.R.S.; GIFFONI, M.G.P; SILVA, L.F.; UTAGAWA, C.Y. Comparative between the PubMed, SciELO and Google Scholar databases with the focus on thematic Medical Education. Cadernos. Volta Redonda: Cadernos UniFOA, n. 28, p. 75-82, 2015.

ROMAGNOLI, F.; NANNI, M.R.; JUNIOR, C.A.S.; SILVA, A.A.; CEZAR, E.; GASPAROTTO, A.C. Predição do Carbono Orgânico do Solo Utilizando Espectroscopia Vis-Nir, Plsr e Regressão Stepwise. Agrarian Academy, Centro Científico Conhecer- Goiânia, v.1, n. 01, p. 251, 2014.

RONQUIM, C.C. Conceitos de fertilidade do solo e manejo adequado para as regiões tropicais. Campinas: Embrapa, 2010.

ROSSITER, D.G. Past, present \& future of information technology in pedometrics. Geoderma, v. 324, p. 131-137, 2018.

SILVA, S.A.; LIMA, J.S.S. Lógica Fuzzy no mapeamento de variáveis indicadoras de fertilidade do solo. Chile: IDESIA, v. 27, n. 3, p. 41-46, 2009. 
SILVA, E.F.; ÁVILA, P.F.; SALGUEIRO, A.R.; CANDEIAS, C.; PEREIRA, H.G. Quantitative-spatial assessment of soil contamination in S. Francisco de Assis due to mining activity of the Panasqueira mine (Portugal). Environmental Science and Pollutition Research, v. 20, p. 534-541, 2013.

STONER, E.R.; BAUMGARDNER, M.F.; WEISMILLER, R.A.; BIEHL, L.L.; ROBINSON, B.F. Extension of laboratory measured soil spectra to field conditions. Soil Science Society of America Journal, v. 44, p. 572, 1980.

SUMFIETH, K. \& DUTTMANN, R., Prediction of soil property distribution in paddy soil landscapes using terrain data and satellite information as indicators. Ecological Indicators, v. 8, n. 5, p. 485-501, 2008

VIEIRA, S.R. Uso de geoestatística em estudos de variabilidade espacial de propriedades do solo. In: Roberto Ferreira Novais; Victor Hugo Alvarez V.; Carlos Ernesto G. R. Schaefer. (Org.). Tópicos em Ciência do Solo. Viçosa: Sociedade Brasileira de Ciência do Solo, v. I, p. 1-54, 2000.
WEI, J.B.; XIAO, D.N.; ZEG, H.; FU, Y.K. Spatial variability of soil properties in relation to land use and topography in a typical small watershed of the black soil region, northeastern China. Environmental Geology, v. 53, n. 8, p. 1663-1672, 2007.

ZONTA, J.H.; BRANDÃO, Z.N.; MEDEIROS, J.C.; SANA, R.S.; SOFIATTI, V. Variabilidade espacial da fertilidade do solo em área cultivada com algodoeiro no cerrado do Brasil. Campina Grande: Revista Brasileira de Engenharia Agrícola, v.18, n. 6, p. 509-602, 2014.

Submetido em 13 de maio de 2019 Aceito em 14 de janeiro 2020 